\title{
Effects of Electroacupuncture on Experimental Periodontitis in Rats
}

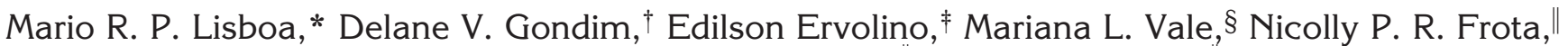 \\ Nara L. T. Nunes, * Viviane C. Mariguela, "Mario Taba Jr., Michel R. Messora, "Flávia A. C. Furlaneto\|
}

Background: Acupuncture has shown the capability of modulating the immuno-inflammatory response of the host. This study aims to evaluate the effects of electroacupuncture (EA) on ligature-induced periodontitis in rats.

Methods: Thirty-two animals were divided into four groups: 1) control; 2) experimental periodontitis (EP); 3) sham-treated (EP/EA-sham); and 4) treated with EA (EP/EA). For the EP groups, a ligature was placed around the right mandibular first molars at day 1. Sessions of EA or EA-sham were assigned every other day. For EA treatment, large intestine meridian points LI4 and LI1 1 and stomach meridian points ST36 and ST44 were used. EA-sham was performed in off-meridian points. Animals were euthanized at day 11 . Histomorphometric and microtomographic analyses were performed. Immunolabeling patterns for the receptor activator of nuclear factor $\kappa \mathrm{B}$ ligand (RANKL), osteoprotegerin (OPG), and tartrate-resistant acid phosphatase (TRAP) were assessed. Expressions of interleukin (IL)- $1 \beta$, matrix metalloproteinase (MMP)-8, IL-6, and cyclooxygenase (COX)-2 messenger RNAs (mRNAs) were evaluated by quantitative reverse transcription-polymerase chain reaction. Data were analyzed statistically ( $P<0.05$, analysis of variance).

Results: Histomorphometric and microtomographic analyses demonstrated that group EP/EA presented reduced alveolar bone loss when compared to group EP $(P<0.05)$. Reduced RANKL immunolabeling and fewer TRAP-positive multinucleated cells were observed in the EA-treated group in relation to group EP. No differences were observed in OPG expression among groups. EA treatment decreased the genic expression of IL-1 $\beta$ and MMP-8 $(P<0.05)$, increased the mRNA expression of IL-6 $(P<0.05)$, and did not modify the genic expression of COX-2 in animals with EP $(P>0.05)$.

Conclusion: It can be concluded that EA reduced periodontal tissue breakdown and the expression of some proinflammatory mediators and a proresorptive factor in EP in rats. J Periodontol 2015;86:801-811.

\section{KEY WORDS}

Bone resorption; electroacupuncture; inflammation mediators; periodontitis.

\footnotetext{
* Department of Clinical Dentistry, Federal University of Ceará, Fortaleza, Ceará, Brazil. † Department of Morphology, Faculty of Medicine, Federal University of Ceará

‡ Department of Basic Sciences, Division of Histology, Dental School of Aracatuba, São Paulo State University, Aracatuba, São Paulo, Brazil.

$\S$ Department of Physiology and Pharmacology, Faculty of Medicine, Federal University of Ceará

II Department of Oral and Maxillofacial Surgery and Periodontology, Ribeirao Preto School of Dentistry, University of São Paulo, Ribeirao Preto, São Paulo, Brazil.

II Department of Veterinary Pathology, São Paulo State University, Jaboticabal, São Paulo, Brazil.
}

A lthough it is known that microbial challenge is necessary for the development of periodontitis, the inflammatory response of the host is ultimately responsible for the appearance of its main clinical features, such as bone loss and periodontal tissue collapse. ${ }^{1}$ Therefore, researchers have been focusing on the modulation of the inflammatory response of the host as a new periodontal approach. ${ }^{2,3}$

Acupuncture is a modality of traditional Chinese medicine that relies on skin stimulation of specific points, called acupoints, by needles. It is considered an adjunct or acceptable alternative treatment for a number of clinical conditions, such as asthma, fibromyalgia, low back pain, carpal tunnel syndrome, headache, menstrual cramps, tennis elbow, stroke rehabilitation, and addiction. ${ }^{4}$ The stimulation of acupoints leads to neuroendocrine responses through many pathways, activating different systems, such as opioid, endocannabinoid, and autonomous nervous systems. ${ }^{5-7}$

It has been proven that acupuncture reduces the genic expression and protein levels of certain inflammatory cytokines, such as interleukin (IL)-1 $\beta$, impairing proinflammatory reactions in arthritis and skin inflammation. ${ }^{7,8}$ It has been used as a treatment for the inflammatory aspects of many experimental diseases, such as colorectal distention, neuropathic pain, and asthma. ${ }^{9-11}$ In addition, it was demonstrated that electroacupuncture (EA) was capable of influencing bone

doi: $10.1902 /$ jop.2015.140630 
metabolism experimentally, enhancing cellular proliferation and bone mineral density (BMD). ${ }^{12,13}$

To the best of the authors' knowledge, there are no experimental studies analyzing the influence of acupuncture treatment on periodontitis. Because acupuncture has been reported as a therapy capable of modulating the inflammatory response,, 14 the purpose of this study is to evaluate the effects of EA on ligature-induced periodontitis in rats.

\section{MATERIALS AND METHODS}

\section{Experimental Model}

This study was conducted in compliance with the ethical principles of the Universal Declaration of Animal Rights by United Nations Educational, Scientific and Cultural Organization. The present study was conducted after review and approval by the Ethics Committee on Animal Research at the Federal University of Ceará, Ceará, Brazil (protocol 56/2012).

Thirty-two adult male rats (Rattus norvegicus albinus, Wistar), weighing 200 to $250 \mathrm{~g}$, were used (Central Animal Facility, Federal University of Ceará). The rats were kept in a 12-hour light/dark cycle with the temperature between $22^{\circ} \mathrm{C}$ and $24^{\circ} \mathrm{C}$. The animals were housed in plastic cages and fed with a selected solid diet and water ad libitum. They were assigned randomly to one of four experimental groups ( $\mathrm{n}=8)$ : 1) control $(\mathrm{C}) ; 2)$ experimental periodontitis (EP); 3) sham-treated (EP/EA-sham); and 4) treated with EA (EP/EA).

\section{Induction of EP}

All animals were anesthetized by an intramuscular injection of ketamine ${ }^{\#}$ (70 mg/kg body weight) and xylazine** (6 mg/kg body weight). A cotton ligature was placed around their right mandibular first molars (M1s), except for the rats of group C, as described by Foureaux et al. ${ }^{2}$ The ligatures were knotted at the buccal surface of the tooth and remained in place for 11 days.

\section{EA and EA-Sham Procedures}

The animals were not anesthetized or sedated for these procedures. To reduce the animals' stress during these events, they were adapted to a specially manufactured bed that allows the exposure of their tails and forepaws and hindpaws. During the 5 days that preceded the EP induction, the rats remained in these beds for 10 minutes/day.

EA and EA-sham were performed with $0.18 \times 8-\mathrm{mm}$ stainless steel needles ${ }^{\dagger \dagger}$ inserted to a depth of $3 \mathrm{~mm}$ under the skin. The acupuncture point selection was based on Traditional Chinese Medicine meridian theory. ${ }^{15}$ In group EP/EA, the large intestine meridian points LI4 and LI11 and the stomach meridian points ST36 and ST44 were stimulated. ${ }^{7,14}$ The stimulation of these acupoints causes a local acute inflammation that targets the activation of opioid, sympathetic,
Table I.

\section{Histometric Analysis of ANBL}

\begin{tabular}{ll}
\hline Experimental Group & ANBL $\left(\mathrm{mm}^{2}\right)$ \\
\hline C & $0.096 \pm 0.022^{\mathrm{a}}$ \\
EP & $0.313 \pm 0.084^{\mathrm{b}}$ \\
EP/EA-sham & $0.324 \pm 0.093^{\mathrm{b}}$ \\
EP/EA & $0.158 \pm 0.057^{\mathrm{a}}$ \\
\hline
\end{tabular}

Mean \pm SD of the area of no bone or periodontal ligament (ANBL) in the furcation region of $M 1 \mathrm{~s}$, with comparisons among groups. Same letters indicate no significant differences among groups $(P<0.05$, ANOVA, Tukey test).

parasympathetic, and endocannabinoid systems, ${ }^{5-7}$ related to anti-inflammatory effects. ${ }^{7,14}$ In group EP/ EA-sham, two sham points located $5 \mathrm{~mm}$ laterally and $5 \mathrm{~mm}$ above the gallbladder meridian point GB30 were stimulated instead. ${ }^{7,14}$ When sham points are stimulated, although a local inflammatory response takes place, the activation of the aforementioned antiinflammatory pathways is not observed. ${ }^{16}$ The LI4 point is located in the forepaw, between the first and second metacarpal bones. The LI11 point is the depression formed when the elbow is flexed at the lateral end of the transverse cubital crease near the lateral epicondyle of the humerus. The ST36 point is at the proximal one-fifth site of the craniolateral surface of the leg distal to the head of the tibia in a depression between the muscles of the cranial tibia and long digital extensor. The ST44 point is at the dorsum of the hindleg, proximal to the web margin between the second and third metatarsals. ${ }^{7,14}$ The GB30 point is located at the junction of the lateral one-third and medial two-thirds distance between the prominence of the greater trochanter and the hiatus of the sacrum. ${ }^{8}$

Sessions of EA and EA-sham were performed every other day at 8:00 am, starting from the day after the ligature placement. All points were stimulated bilaterally and simultaneously with low-frequency and rectangular pulses (frequency $=10 \mathrm{~Hz}$; recurrence time $=1$ second; intensity $=3 \mathrm{~mA})^{7,14}$ during 20 minutes, using an electric stimulation device. ${ }^{\ddagger}$ EA and EA-sham procedures were conducted by an experienced practitioner (DVG). ${ }^{7,14,17}$

The animals were euthanized under anesthesia with a solution of xylazine ( $30 \mathrm{mg} / \mathrm{kg}$ body weight) and ketamine ( $240 \mathrm{mg} / \mathrm{kg}$ body weight) 11 days after the placement of the ligatures. Samples of gingival tissues around the right M1s of each animal were

\# Dopalen, Agribrands, Paulinia, São Paulo, Brazil.

** Rompum, Bayer Saude Animal, Sao Paulo, São Paulo, Brazil.

†† Dongbang, Dongbang AcuPrime, Ungcheon, Korea

‡ NKL EL 530, NKL Produtos Eletrônicos, Brusque, Santa Catarina, Brazil. 
Table 2.

\section{Histopathologic Analysis of the Furcation Region of M1s: Distribution of the Animals per} Score Evaluated

\begin{tabular}{|c|c|c|c|c|}
\hline \multirow[b]{2}{*}{ Parameters and Respective Scores } & \multicolumn{4}{|c|}{ Experimental Groups ( $\mathrm{n}=8$ animals/score) } \\
\hline & C & EP & EP/EA-sham & EP/EA \\
\hline \multicolumn{5}{|l|}{ Intensity of local inflammatory infiltrate } \\
\hline (0) Absence of inflammation & 8 & 0 & 0 & 0 \\
\hline (I) Small amount of inflammatory cells & 0 & 0 & 0 & 7 \\
\hline $\begin{array}{l}\text { (2) Moderate amount of inflammatory } \\
\text { cells }\end{array}$ & 0 & 6 & 6 & I \\
\hline (3) Large amount of inflammatory cells & 0 & 2 & 2 & 0 \\
\hline \multicolumn{5}{|l|}{ Extension of inflammatory infiltrate } \\
\hline (0) Absence of inflammation & 8 & 0 & 0 & 0 \\
\hline $\begin{array}{l}\text { (I) Extending to part of the CT of the } \\
\text { furcation area }\end{array}$ & 0 & 0 & । & 8 \\
\hline $\begin{array}{l}\text { (2) Extending to the whole CT of the } \\
\text { furcation area }\end{array}$ & 0 & 7 & 6 & 0 \\
\hline $\begin{array}{l}\text { (3) Extending to the whole } \mathrm{CT} \text { and to the } \\
\text { bone tissue of the furcation area }\end{array}$ & 0 & 1 & । & 0 \\
\hline \multicolumn{5}{|l|}{$\begin{array}{l}\text { External root resorption (cementum and } \\
\text { dentin) }\end{array}$} \\
\hline (0) Absent & 8 & 0 & 0 & 4 \\
\hline (I) Only inactive resorption areas & 0 & 0 & 0 & 2 \\
\hline (2) Few active resorption areas & 0 & 7 & 8 & 2 \\
\hline (3) Many active resorption areas & 0 & 1 & 0 & 0 \\
\hline \multicolumn{5}{|l|}{ Alveolar bone resorption } \\
\hline (0) Within normality patterns & 8 & 0 & 0 & 0 \\
\hline (I) Small amount of resorption areas & 0 & 0 & 0 & 8 \\
\hline (2) Moderate amount of resorption areas & 0 & 0 & I & 0 \\
\hline (3) Large amount of resorption areas & 0 & 8 & 7 & 0 \\
\hline \multicolumn{5}{|l|}{ CT pattern } \\
\hline $\begin{array}{l}\text { (0) Moderate amount of fibroblasts and } \\
\text { large amount of collagen fibers (dense CT) }\end{array}$ & 8 & 0 & 0 & 3 \\
\hline $\begin{array}{l}\text { (I) Moderate amount of both fibroblasts } \\
\text { and collagen fibers }\end{array}$ & 0 & 0 & 0 & 5 \\
\hline $\begin{array}{l}\text { (2) Small amount of both fibroblasts and } \\
\text { collagen fibers; presence of interstitial } \\
\text { edema }\end{array}$ & 0 & 8 & 8 & 0 \\
\hline $\begin{array}{l}\text { (3) Severe tissue breakdown with } \\
\text { interstitial edema and necrotic areas }\end{array}$ & 0 & 0 & 0 & 0 \\
\hline \multicolumn{5}{|l|}{ Alveolar bone pattern } \\
\hline $\begin{array}{l}\text { (0) Bone trabeculae with regular contour } \\
\text { coated with active osteoblasts, including } \\
\text { areas of new bone formation }\end{array}$ & 8 & 0 & 0 & 2 \\
\hline $\begin{array}{l}\text { (I) Bone trabeculae with irregular contour } \\
\text { coated with active osteoblasts and } \\
\text { osteoclasts }\end{array}$ & 0 & 0 & 0 & 6 \\
\hline $\begin{array}{l}\text { (2) Bone trabeculae with irregular contour } \\
\text { coated with active osteoclasts }\end{array}$ & 0 & 7 & 8 & 0 \\
\hline $\begin{array}{l}\text { (3) Areas of necrotic bone and bone } \\
\text { trabeculae with irregular contour coated } \\
\text { with active osteoclasts }\end{array}$ & 0 & 1 & 0 & 0 \\
\hline
\end{tabular}

$\mathrm{CT}=$ connective tissue. 
collected and stored at $-80^{\circ} \mathrm{C}$. The right mandibles were excised, fixed in $4 \%$ paraformaldehyde for 24 hours, and rinsed with water.

\section{Microcomputed Tomography Analyses}

Non-demineralized specimens were scanned by a cone-beam microcomputed tomography (micro-CT) system. $\S \S$ The $\mathrm{x}$-ray generator was operated at an accelerated potential of $50 \mathrm{kV}$ with a beam current of $200 \mu \mathrm{A}$ and an exposure time of 650 milliseconds/ projection. Images were produced with a voxel size of $6 \times 6 \times 6 \mu \mathrm{m}$.

Using appropriate software, |||| the generated threedimensional models were rotated into a standard position according to the following criteria: 1) in the transaxial plane, the M1 had its axis positioned vertically; and 2) in the coronal plane, the mandibular bone was oriented vertically, with the mesial root of the M1 in the upper portion of the image. Linear measurements on alveolar bone level (ABL) were performed at three different sites: 1) buccal; 2) lingual; and 3) interproximal. For buccal and lingual sites, on the transaxial image passing through the distal root of the M1, the linear distances from the cemento-enamel junction (CEJ) to the buccolingual alveolar bone crest $(A B C)$ were measured. For the interproximal site, the coronal dataset was analyzed using appropriated software. II The distance between the last image, showing the $A B C$ between the mandibular second molar and $M 1$, and the first image, showing the CEJ of $M 1$, was measured.

BMD was also analyzed. A volume of interest (prismatic section) was outlined from the apexes of all roots of $M 1$ up to the roof of the furcation of M1, touching the root surfaces, in all images of the coronal dataset. BMD was determined by comparing the volume of interest of the samples with a pattern that presented a known mineral density, using the same software applied for the analysis of the interproximal site.

All micro-CT analyses were performed by one masked and calibrated examiner (MRPL).

\section{Histopathologic and Histometric Analyses}

The specimens were decalcified in 10\% EDTA solution. After complete decalcification, they were processed and embedded in paraffin. Serial sections, $5 \mu \mathrm{m}$ thick, were obtained in a mesio-distal direction. The sections were stained with hematoxylin and eosin. Sections representing the most central bucco-lingual portion in the furcation area of the right $M 1 \mathrm{~s}$ were selected for histopathologic and histometric analyses (Tables 1 and 2 ). The histopathologic analysis was performed by a certified histologist (EE) using a light microscope. \#\# The parameters analyzed were based on the study by Furlaneto et al., ${ }^{3}$ and the scores were adapted from the study by Gomes-Filho et al. ${ }^{18}$ (Table 2 ).

For histometric analysis, photomicrographs were captured using a digital camera*** connected to a light microscope $\mathrm{e}^{\dagger \dagger}$ with an original magnification of $\times 40$. The images were analyzed using appropriate

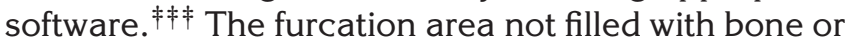
periodontal ligament (PDL) (area of no bone or PDL [ANBL]) was measured by outlining the region surrounded by the roof of the furcation, the most coronal portion of the $A B C$ in the furcation, and the mesial and distal roots of the M1. Histometric analysis was performed by one masked and calibrated examiner (NPRF).

\section{Immunohistochemical Analyses}

Immunohistochemical processing was performed through the indirect immunoperoxidase method, as described previously. ${ }^{3}$ The histologic slides containing samples from all the experimental groups were divided into three batches, and each batch was incubated with one of the following primary antibodies: 1) goat antitartrate-resistant acid phosphatase (TRAP);§§§ 2) goat anti-receptor activator of nuclear factor- $\kappa \mathrm{B}$ ligand (RANKL); ; IIII or 3) goat anti-osteoprotegerin (OPG). . ศศศा

Three histologic sections from each animal were evaluated under light microscopy with an optical microscope $\mathrm{e}^{\# \# \#}$ by one masked and calibrated examiner (EE). A quantitative immunolabeling analysis was performed for TRAP-positive cells, distinguished as TRAP-positive multinucleated cells (mature osteoclasts) and TRAP-positive mononuclear cells. ${ }^{3}$ TRAP-positive cells were counted in areas of 2,000 $\times 2,000 \mu \mathrm{m}$ in the central part of the furcation septum at $\times 200$ magnification. The coronal limit of the area was the ABC, which extended apically for a distance of $2,000 \mu \mathrm{m}$. Semiquantitative analyses of the immunolabeling of RANKL and OPG were conducted in the entire furcation region at $\times 400$ magnification. The coronal limit of this area was the roof of furcation, from which the region analyzed extended apically, comprising PDL and alveolar bone. The immunolabeling scores were determined as follows: score 0 , no immunolabeling (total absence of immunoreactivity in the area); score 1, low immunolabeling pattern (approximately equal to onequarter of the area presenting immunoreactivity); score 2, moderate immunolabeling pattern (approximately equal to one-half of the area presenting immunoreactivity); score 3, high immunolabeling pattern (approximately equal to three-quarters of the

$\S \S$ Skyscan 1172, Bruker, Kontich, Belgium

III Data Viewer v.1.5.0, Bruker.

II CT-Analyser v.1.13.5.1+, Bruker.

\#\# AxioVision v.4.8.2, Carl Zeiss, Jena, Germany.

* * * DFC295, Leica Microsystems, Wetzlar, Germany.

$\dagger \dagger \dagger$ DM2000, Leica Microsystems.

$\neq \neq \neq$ NIH ImageJ, National Institutes of Health, Washington, DC.

$\S \S \S 1: 100$ goat anti-TRAP, sc-30833, Santa Cruz Biotechnology, Santa Cruz, CA.

|l|| 1:100 goat anti-RANKL, sc-7628, Santa Cruz Biotechnology.

ข1ๆ $1: 100$ goat anti-OPG, sc-8468, Santa Cruz Biotechnology.

\#\#\# AxioVision v.4.8.2, Carl Zeiss. 


\section{Micro-CT Analyses}

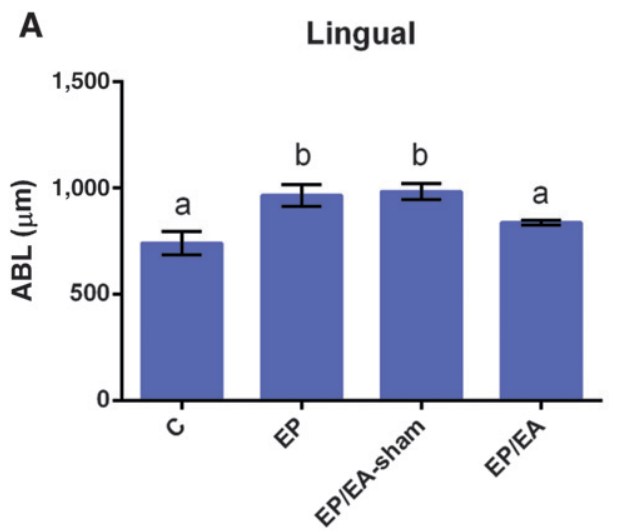

B

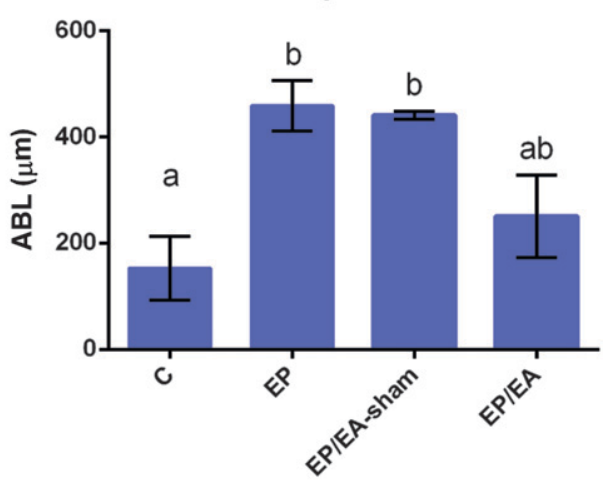

C

Buccal

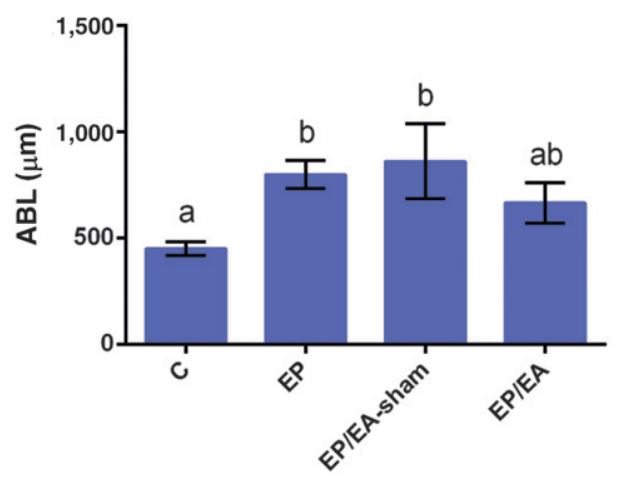

D

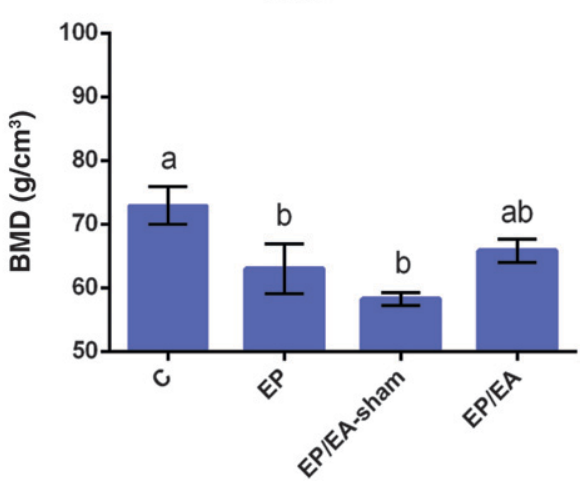

Figure I.

Micro-CT analyses. Means and standard deviations of the ABL of the specimens with comparisons between groups at lingual (A), interproximal (B), and buccal (C) sites and of the BMD assessment (D). Same letters indicate no significant differences between groups ( $\mathrm{P}<0.05$, ANOVA, Tukey test).

area presenting immunoreactivity); and score 4, extremely high immunolabeling pattern (almost the totality of the area presenting immunoreactivity).

\section{Quantitative Reverse Transcription-Polymerase Chain Reaction}

The gingival samples were macerated under freezing with liquid nitrogen. The tissues were homogenized with RNA extraction reagent**** (1 mL/0.1 mg gingival tissue) as recommended by the protocol of the manufacturer. Total RNA extraction was performed by an extraction kit ${ }^{\dagger \dagger \dagger}$ following the recommendations of the manufacturer and was

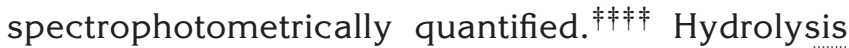

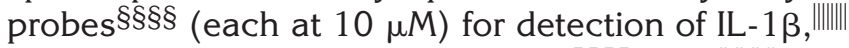

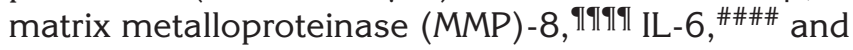
cyclooxygenase (COX) $-2 * * * * *$ and $5 \mu \mathrm{L}$ complementary DNA were used in every reaction. The amplification was performed in a thermocycler ${ }^{\dagger \dagger \dagger \dagger \dagger}$ for 40 cycles and according to the protocol of the manufacturer. For messenger RNA (mRNA) analysis, the genic expression levels of IL-1 $\beta$, MMP-8, IL-6, and COX-2 were calculated by comparison with levels of $\beta$ actin mRNA expression in the same sample, using the cycle threshold method $(\Delta \Delta \mathrm{CT})$. The cycle thresholds of the target genes were normalized to an endogenous reference ( $\beta$-actin) and relativized to a calibrator group (group C), and the relative genic expression was given by the $\Delta \Delta C T$ method using equation $2^{-\Delta \Delta C T}$ in the study by Livak and Schmittgen. ${ }^{19}$

\section{Examiner Calibration}

All analyses were performed by masked examiners (MRPL, EE, NPRF). To estimate the intraexaminer error, the same sample was measured again 1 week after the first measurement. For continuous variables, the calibrations of the examiners were assessed by intraclass correlation coefficient $(>0.6)$. Weighted Cohen $\kappa$ was used to determine the examiner calibration in the analyses of the immunolabeling of RANKL and OPG (>90\%).

\section{Statistical Analyses of the Data}

Data were grouped and presented as means and standard deviations (continuous variables) or medians, interquartile ranges, and maximum and minimum values (ordinal variables). Normality and homoscedasticity of the data were verified. The significance of differences among groups in relation to the immunolabeling pattern of RANKL and OPG was determined by Kruskal-Wallis tests, followed by Dunn multiple comparison post hoc test. The data from the other analyses were assessed by analysis of variance (ANOVA), followed by post hoc Tukey test. The significance level was set at $5 \%$ in all tests.

\footnotetext{
**** TRIzol, Invitrogen, Life Technologies, Thermo Fisher Scientific, Waltham, MA.

†††† SV Total RNA Isolation System, Promega, Madison, WI.

㤄住 NanoVue Plus, GE Healthcare, Fairfield, CT.

$\S \S \S \S$ TaqMan Gene Expression Assays, Life Technologies, Thermo Fisher Scientific.

Rn00580432_m1, Life Technologies, Thermo Fisher Scientific.

ๆๆ|ๆ Rn00573646_m1, Life Technologies, Thermo Fisher Scientific

\#\#\#\# Rn01410330_m1, Life Technologies, Thermo Fisher Scientific.

***** Rn01483828_m1, Life Technologies, Thermo Fisher Scientific.

$\dagger \dagger \dagger \dagger \dagger$ StepOnePlus, Applied Biosystems, Thermo Fisher Scientific.
} 

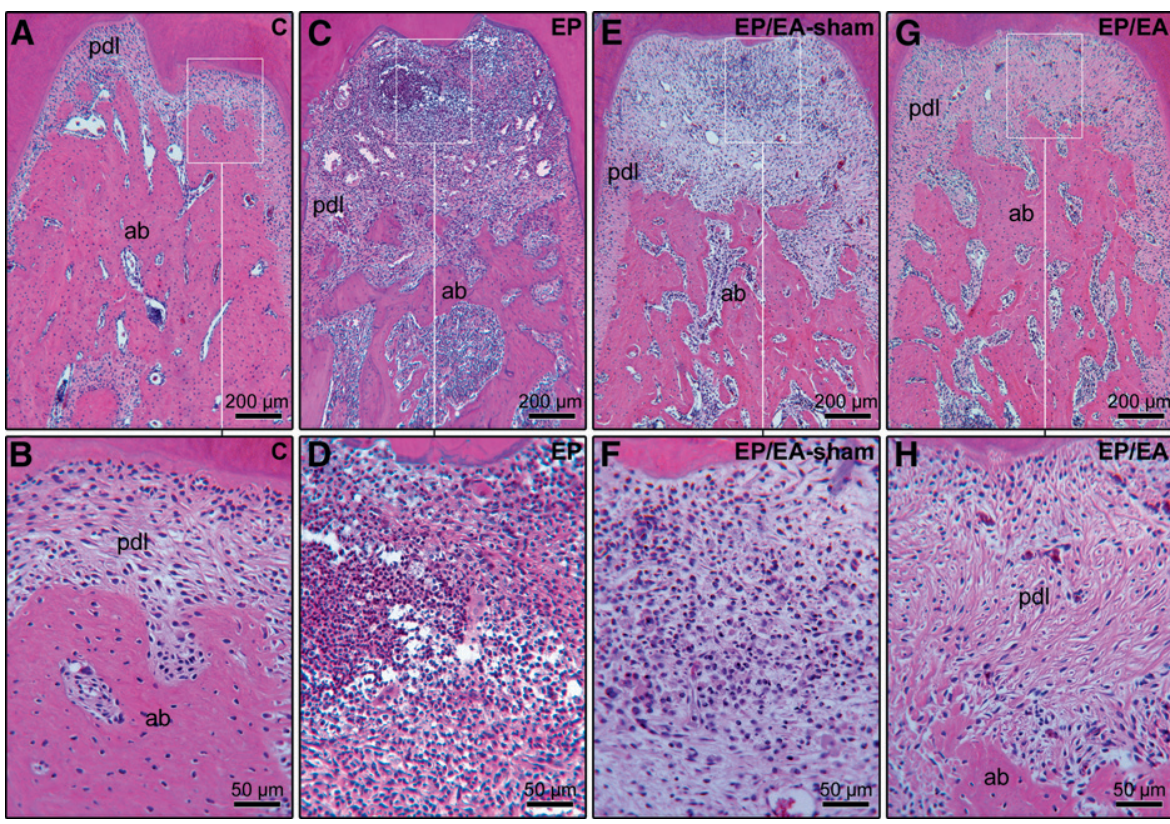

Figure 2.

Photomicrographs of periodontal tissues in the furcation areas of M Is. $\boldsymbol{A}$ and $\mathbf{B}) \mathrm{Group} C ; \boldsymbol{C}$ and $\mathbf{D}) E$. E and F) EPIEA-sham. G and $\boldsymbol{H}$ ) EP-EA. ab = alveolar bone; pdl = periodontal ligament. (Hematoxylin and eosin stain.)

\section{RESULTS}

All animals tolerated the experimental procedures well and remained healthy throughout the experimental period.

\section{Micro-CT Analyses}

At the lingual site, group EP/EA presented significantly less alveolar bone resorption than groups EP and EP/ EA-sham $(P<0.05)$ and no significant difference when compared with group $C(P>0.05)$ (Fig. 1A). However, at both the buccal and interproximal sites, group EP/ EA demonstrated ABL not statistically different from the other groups $(P>0.05)$ (Figs. 1B and 1C).

$\mathrm{BMD}$ assessment revealed that group EP/EA presented a reduced $B M D$ when compared to group $C$ but a greater BMD in relation to groups EP and EP/EAsham, although no significant differences were found when group EP/EA was compared to any of the other groups $(P>0.05)$ (Fig. 1D).

\section{Histopathologic Analyses}

The results of the histopathologic analysis based on scores are depicted in Table 2. In group C, PDL presented a great amount of collagen fibers, fibroblasts, and blood vessels. Collagen fibers were inserted in both cementum and alveolar bone. The cementum surface was totally sound and covered with cementoblasts. The bone tissue of the interradicular septum presented a few irregularities on its surface, and it was coated with osteoblasts or bone lining cells. At this site, bone trabeculae were considerably thick, limiting small medullary spaces

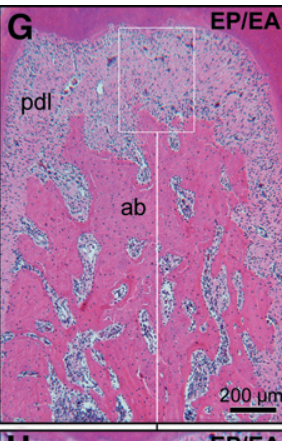

(Figs. 2A and 2B). Groups EP and EP/EA-sham presented a moderate inflammatory infiltrate predominantly composed of mononuclear cells and a small amount of neutrophils in the PDL. This ligament presented interstitial edema and a reduced amount of collagen fibers when compared with the PDL observed in group C. In the majority of the specimens, the cementum presented small areas of active resorption. The bone tissue in the interradicular septum presented thin trabeculae, with a very irregular contour and many active osteoclasts (Figs. 2C through 2F). In group EP/EA, the mononuclear inflammatory infiltrate in PDL was very slight. This tissue was much more fibrous and less edematous than PDL present in groups EP and EP/EA-sham, which demonstrates minor alterations in collagen fibrilogenesis. The cementum was sound in the majority of the specimens, although some samples presented areas with active resorption. Bone tissue in the interradicular septum comprised bone trabeculae with an irregular external contour, and it was covered with osteoblasts or bone lining cells. Few active osteoclasts were observed (Figs. $2 \mathrm{G}$ and $2 \mathrm{H}$ ).

\section{Histometric Analyses}

Group EP/EA presented lower ANBL than groups EP $(P<0.05)$ and EP/EA-sham $(P<0.05)$. There were no differences in ANBL when groups $C$ and EP/EA were compared $(P>0.05)$ (Table 1$)$.

\section{Immunohistochemical Analyses}

The results of immunohistochemical analyses and photomicrographs showing immunolabeling are presented in Figures 3 and 4, respectively. The amount of TRAP-positive multinucleated cells was significantly greater in groups EP (Figs. 3A and 4B) and EP/ EA-sham (Fig. 4C) when compared with group $C$ $(P<0.05)$ (Fig. 4A). Group EP/EA (Fig. 4D) presented less TRAP-positive multinucleated osteoclasts than group EP $(P<0.05)$. There were no significant differences in the number of TRAP-positive mononuclear cells among the groups $(P>0.05)$ (Fig. 3B).

Groups EP (Fig. 4F) and EP/EA-sham (Fig. 4G) presented higher immunolabeling patterns for RANKL than groups $C$ (Fig. 4E) and EP/EA $(P<0.05)$ (Figs. $3 \mathrm{C}$ and $4 \mathrm{H})$. The OPG immunolabeling pattern was similar in all groups, ranging from low to moderate $(P>0.05)$ (Figs. 3D and 4I through 4L). 
Immunohistochemical Analyses

A

TRAP-Positive Multinucleated Cells
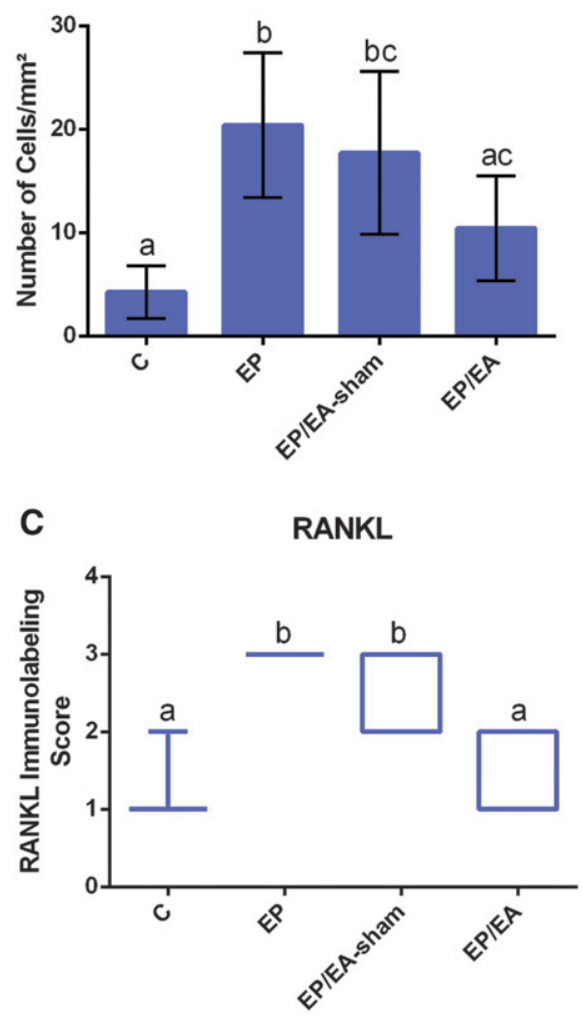

B TRAP-Positive Mononuclear Cells

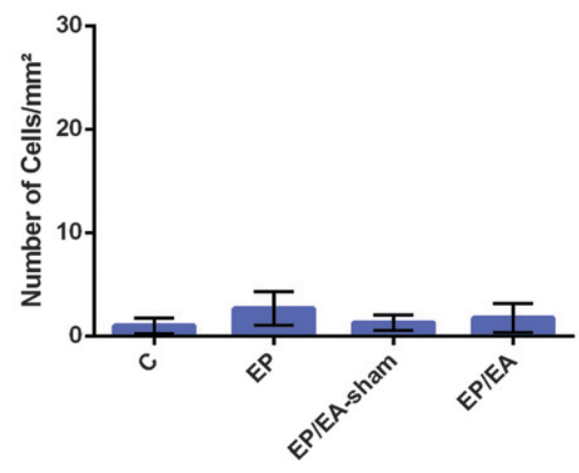

D OPG

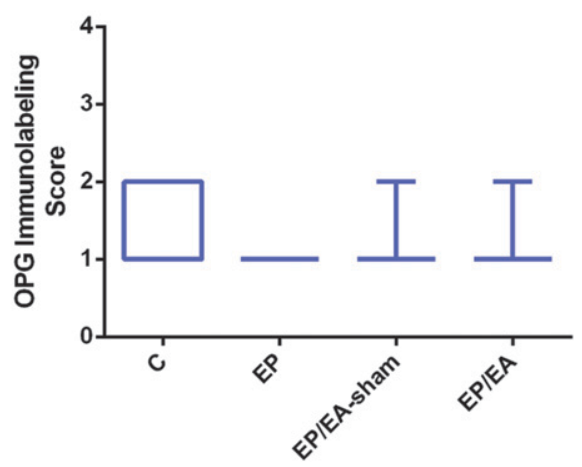

Figure 3.

Immunohistochemical analyses. Means and standard deviations of the number of TRAP-positive multinucleated (A) and mononuclear (B) cells, with comparison between groups. Same letters indicate no significant differences between groups ( $P<0.05 ; A N O V A$, Tukey test). Medians, interquartile ranges, and maximum and minimum values of the immunolabeling scores for RANKL (C) and OPG (D). Same letters indicate no significant differences between groups ( $P<0.05$; Kruskal-Wallis, Dunn tests).

\section{Reverse Transcription-Polymerase Chain Reaction Analyses}

Groups EP/EA and EP/EA-sham presented lower levels of IL-1 $\beta$ mRNA when compared with group EP (5.4-fold higher when compared with group C; $P<0.05)$ and levels similar to the ones presented by group $C(P>0.05)$ (Fig. 5A).

Group EP/EA presented a decreased expression of MMP-8 mRNA when compared with groups EP (3.47fold higher in relation to group $C ; P<0.05)$ and $E P / E A-$ sham (2.08-fold higher when compared with group $C$; $P<0.05)$. Conversely, the expression of MMP-8 mRNA in group EP/EA was similar to the one found in animals not submitted to the induction of EP $(P>0.05)$ (Fig. 5B).

Group $C$ presented higher expression of IL- 6 mRNA than group $\mathrm{EP}(P<0.05)$. The animals from group EP/EA exhibited expression of IL- 6 mRNA even greater than that presented by group $C(1.30$-fold higher; $P<0.05)$ (Fig. 5C).
Group EP/EA-sham presented increased expression of COX-2 mRNA when compared with the other groups (2.11-fold higher compared with group $C$; $P<0.05$ ) (Fig. 5D).

\section{DISCUSSION}

Notably, the present model of periodontitis induction was effective. Significant bone loss, decrease in $\mathrm{BMD}$, and moderate inflammatory infiltrate in the PDL were observed in group EP but not in group C. Genic expression of some proinflammatory molecules, such as IL-1 $\beta$ and MMP-8, presented upregulation in group EP when compared with group C. These mediators are commonly associated with periodontitis. ${ }^{20,21}$ In fact, authors have shown that the ligature model is one of the most representative experimental models of periodontitis. ${ }^{22}$

Overall, the treatment with EA was able to decrease bone resorption in EP in the present study. It was noticeably observed when the bone tissue was analyzed at furcation and lingual sites (histologically and through microCT analysis, respectively). Although group EP/EA did not present significant differences in ABL when micro-CTs of interproximal and buccal sites were analyzed, a clear trend toward a reduction in bone resorption was observed in this group when compared with group EP. The same was noticed in relation to the $B M D$ measured at the furcation area of the specimens. In fact, it has been shown that EA positively influenced bone metabolism. Radiographic analysis of ovariectomized rabbits' femurs demonstrated that EA treatment was capable of restoring their BMD toward what was observed in naive rabbits. ${ }^{23}$ In ovariectomized rats, EA prevented osteoporosis, enhancing the number of bone trabeculae and the trabecular area. $^{12}$

To the best of the authors' knowledge, this study demonstrates the first evidence that EA may modulate the expression of RANKL, because its immunohistochemical expression was reduced in the treated group. RANKL binds to RANK, leading to osteoclastic differentiation. ${ }^{24}$ The reduction in the RANKL immunolabeling pattern observed in group 


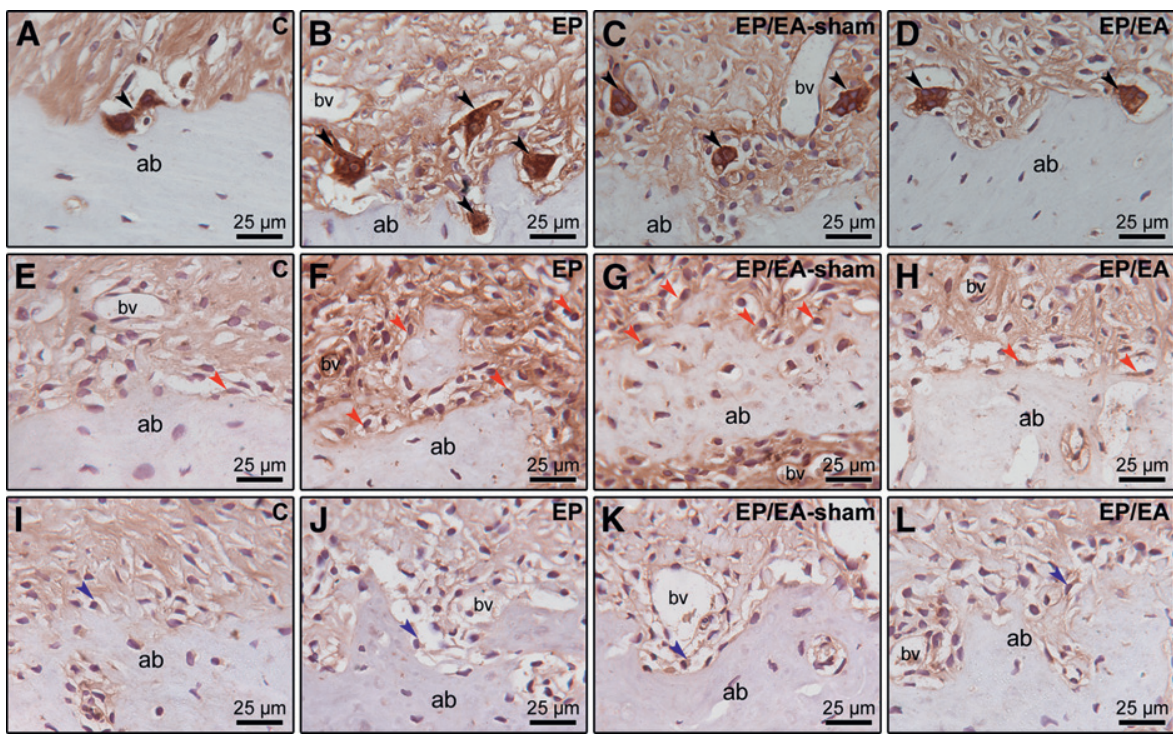

Figure 4.

Photomicrographs showing immunolabeling for TRAP (A through $\boldsymbol{D})$, RANKL (E through $\boldsymbol{H})$, and $O P G$ (I through $L$ ) in the furcation areas of $M$ Is. A, E and I) Group C. B, F, and J) EP. C, G, and K) EP/EAsham. D, $H$ and L) EPIEA. ab = alveolar bone; bv = blood vessel; black arrowheads = TRAP-positive multinucleated cells; blue arrowheads = OPG-positive cells; red arrowheads = RANKL-positive cells. (Hematoxylin counterstaining.)

EP/EA, compared with the untreated animals, might have been responsible for the reduction in the TRAPpositive multinucleated cells found in this group, decreasing alveolar bone resorption. OPG binds to RANKL, blocking its interaction with RANK and reducing osteoclastic differentiation. ${ }^{24}$ However, no differences were observed regarding the expression of OPG among the groups.

In the present study, it was observed that animals treated with EA presented reduced mRNA expression of IL-1 $\beta$ and MMP- 8 when compared with untreated animals. These results are in accordance with other studies that found a significant decrease in the mRNA expression and/or protein levels of IL-1 $\beta$ in experimental wound healing and paw inflammation in rats when they were treated with acupuncture or EA. ${ }^{8,25}$ Acupuncture was capable of reducing mRNA expression levels of MMP-9 and MMP-13. ${ }^{26,27}$ However, to the best of the authors' knowledge, this is the first assessment of the effects of EA on MMP-8.

Compared with group $C$, decreased genic expression of IL- 6 was observed in group EP, and increased genic expression of IL- 6 was observed in group EP/ EA. Although some reported the expression of IL- 6 as an important biomarker of periodontitis progression, ${ }^{28}$ it was hypothesized that IL-6 might have an antiinflammatory role in bone breakdown. ${ }^{29}$ Knock-out mice for the IL- 6 gene presented more severe bone breakdown than wild-type mice in experimental per- iapical lesion. ${ }^{29}$ In fact, Darowish et al. ${ }^{30}$ reported that IL- 6 plays an important role in bone protection, blocking the differentiation of early osteoclastic cells. Therefore, the elevated genic expression of IL-6 observed in group EP/EA in this study might have favored a decrease in bone resorption.

The levels of COX-2 mRNA expression were not statistically different between groups $\mathrm{C}$ and $\mathrm{EP}$. The expression of COX-2 in periodontal tissues is correlated closely to the amount of prostaglandin $E_{2}$ $\left(\mathrm{PGE}_{2}\right) .^{31}$ Although $\mathrm{PGE}_{2}$ was recognized initially for its effects on bone resorption, it became evident that it also stimulates bone formation. ${ }^{31}$ Understanding the role of prostaglandins in skeletal metabolism has been complicated because they act locally and transiently, are regulated at several levels, have multiple receptors, and can have opposing effects depending on the test system. ${ }^{32}$ The treatment with EA does not reduce the COX-2 genic expression when compared with the untreated animals (group EP) in the present study. In fact, although it was found that acupuncture may decrease COX-2 and $\mathrm{PGE}_{2}$ levels, ${ }^{33}$ it was also demonstrated that acupuncture effects might not be mediated by changes in prostaglandin levels. ${ }^{34}$

In studies evaluating the effects of acupuncture, it is essential to carefully select the sites of sham acupuncture. It is possible that the acupoints for other unrelated conditions or non-acupoints on the meridian can also exert a certain degree of therapeutic effects. ${ }^{35}$ Therefore, in the present study, nonacupoints outside the meridian were used for sham acupuncture, as recommended. ${ }^{35}$ Although group EP/EA-sham presented worse results than group EP/ EA in most of the analyses, it is intriguing that group EP/EA-sham presented reduced expressions of IL-1 $\beta$ and MMP-8 mRNAs when compared to group EP. A possible explanation for these results is the fact that the electrical stimulation itself may be conducive to an anti-inflammatory reaction. ${ }^{36-38}$ Transcutaneous electrical nerve stimulation is a non-acupoint electrical stimulation management that has been shown to reduce the production of IL- $1 \beta$ in experimental wound healing in rats. ${ }^{37}$ Despite the lack of evidence regarding the effects of electrical stimulation in genic expression or production of MMP-8, other MMPs, such as MMP-9 and MMP-1, as well as the tissue 


\section{qRT-PCR Analyses}
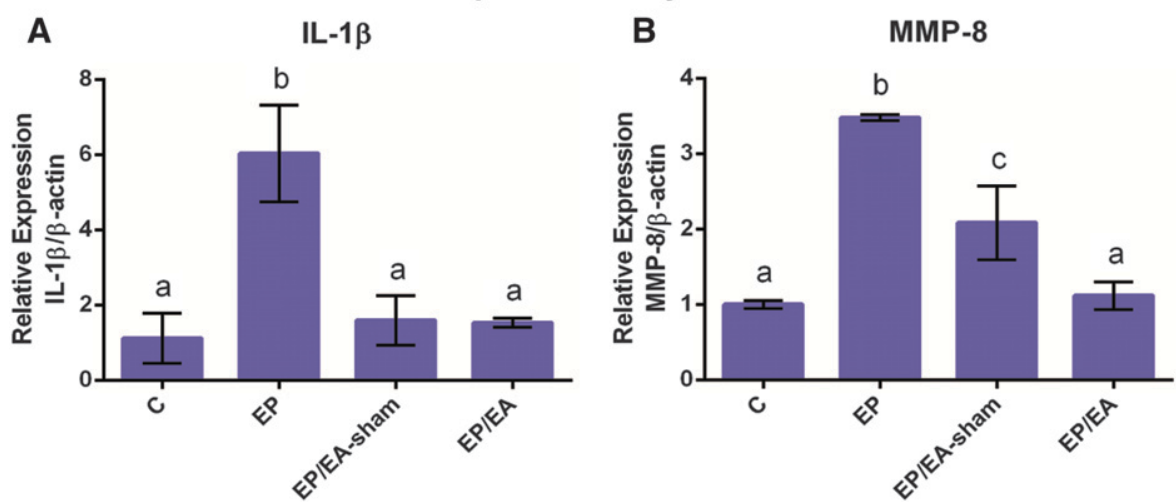

$$
\text { C }
$$

IL-6
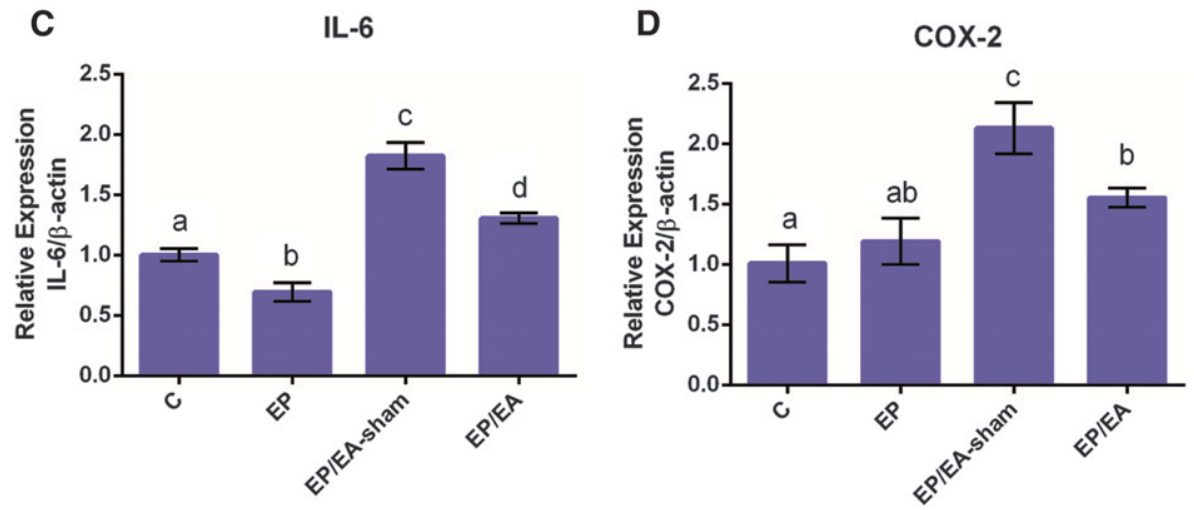

endocannabinoid, sympathetic, and parasympathetic systems. In addition, although the acupoints used in this study have been proved to present antiinflammatory effects, ${ }^{7,14}$ many other acupoints that succeeded in modulating the immunoinflammatory response should be investigated, such as the ones located at the stomach, bladder, gallbladder, and governing vessel meridians. $8,11,40,41$

\section{CONCLUSION}

Within the limits of the present study, it can be concluded that EA reduced periodontal tissue breakdown and the expression of some proinflammatory mediators and a proresorptive factor in EP in rats.

\section{ACKNOWLEDGMENTS}

This work was supported by the National Council for Research and Technological Development Process (150128/2013-4) and Federal University of Ceará, Fortaleza, Ceará, Brazil. This study received the International Association for Dental Research Unilever Hatton Divisional Award

inhibitor of metalloproteinase-1, may be influenced by electrical stimulus. ${ }^{36,38}$ However, it is important to emphasize that the inflammatory process is complex and involves many aspects besides the roles played by IL- $1 \beta$ and MMP-8. This may explain why the decrease in their genic expression did not result in a reduced alveolar bone loss in sham-treated animals in this study.

Besides the fact that this study presents the inherent limitations of experimental research in rats, other investigations should be performed to understand the actual influence of the EA treatment on periodontitis before a clinical application could be projected. The mRNA expressions of some inflammatory mediators are evaluated in this study, but because these assays are not necessarily correlated to the tissue expression of the target molecules, ${ }^{39}$ other analysis should be performed. Additional studies are encouraged to clarify the possible participation of other inflammatory mediators. It is also necessary to elucidate the pathways by which EA influenced some inflammatory aspects in EP, evaluating the role of
(Brazilian Division) in 2014. The authors report no conflicts of interest related to this study.

\section{REFERENCES}

1. Darveau RP. Periodontitis: A polymicrobial disruption of host homeostasis. Nat Rev Microbiol 2010;8:481-490.

2. Foureaux RdeC, Messora MR, de Oliveira LF, et al. Effects of probiotic therapy on metabolic and inflammatory parameters of rats with ligature-induced periodontitis associated with restraint stress. J Periodontol 2014;85:975-983.

3. Furlaneto FA, Nunes NL, Oliveira Filho IL, et al. Effects of locally administered tiludronic acid on experimental periodontitis in rats. J Periodontol 2014;85:1291-1301.

4. National Institutes of Health. Acupuncture. NIH Consensus Statement Online 1997 Nov 3-5; 15:1-34. Available at: http://consensus.nih.gov/1997/1997acupuncture107html. htm. Accessed January 6, 2015.

5. Kavoussi B, Ross BE. The neuroimmune basis of antiinflammatory acupuncture. Integr Cancer Ther 2007;6: 251-257.

6. Kim HW, Uh DK, Yoon SY, et al. Low-frequency electroacupuncture suppresses carrageenan-induced paw inflammation in mice via sympathetic post-ganglionic neurons, while high-frequency EA suppression is 
mediated by the sympathoadrenal medullary axis. Brain Res Bull 2008;75:698-705.

7. Gondim DV, Araújo JC, Cavalcante AL, et al. CB1 and CB2 contribute to antinociceptive and anti-inflammatory effects of electroacupuncture on experimental arthritis of the rat temporomandibular joint. Can $J$ Physiol Pharmacol 2012;90:1479-1489.

8. Su TF, Zhao YQ, Zhang LH, et al. Electroacupuncture reduces the expression of proinflammatory cytokines in inflamed skin tissues through activation of cannabinoid CB2 receptors. Eur J Pain 2012;16: 624-635.

9. Cui KM, Li WM, Gao X, Chung K, Chung JM, Wu GC. Electro-acupuncture relieves chronic visceral hyperalgesia in rats. Neurosci Lett 2005;376:20-23.

10. Lorenzini L, Giuliani A, Giardino L, Calzà L. Laser acupuncture for acute inflammatory, visceral and neuropathic pain relief: An experimental study in the laboratory rat. Res Vet Sci 2010;88:159-165.

11. Xu YD, Cui JM, Wang Y, et al. Proteomic analysis reveals the deregulation of inflammation-related proteins in acupuncture-treated rats with asthma onset. Evid Based Complement Alternat Med 2012;2012:850512.

12. Zhou J, Chen S, Guo H, et al. Electroacupuncture prevents ovariectomy-induced osteoporosis in rats: A randomised controlled trial. Acupunct Med 2012;30:37-43.

13. Inoue $M$, Nakajima $M$, Hojo T, Itoi $M$, Kitakoji H. The effect of electroacupuncture on osteotomy gap healing in a rat fibula model. Acupunct Med 2013;31:222-227.

14. Gondim DV, Costa JL, Rocha SS, Brito GA, Ribeiro RdeA, Vale ML. Antinociceptive and anti-inflammatory effects of electroacupuncture on experimental arthritis of the rat temporomandibular joint. Can J Physiol Pharmacol 2012;90:395-405.

15. Zhou W, Benharash P. Effects and mechanisms of acupuncture based on the principle of meridians. $J$ Acupunct Meridian Stud 2014;7:190-193.

16. Ceniceros S, Brown GR. Acupuncture: A review of its history, theories, and indications. South Med J 1998; 91:1121-1125.

17. Gondim DV, Carvalho KM, Vale ML. Pain behavior to electroacupuncture in rabbit tooth pulp. Braz J Oral Sci 2010;9:415-420.

18. Gomes-Filho JE, Duarte PC, Ervolino E, et al. Histologic characterization of engineered tissues in the canal space of closed-apex teeth with apical periodontitis. $J$ Endod 2013;39:1549-1556.

19. Livak KJ, Schmittgen TD. Analysis of relative gene expression data using real-time quantitative PCR and the 2(-Delta Delta C(T)) Method. Methods 2001;25: 402-408.

20. Page RC, Offenbacher S, Schroeder HE, Seymour GJ, Kornman KS. Advances in the pathogenesis of periodontitis: Summary of developments, clinical implications and future directions. Periodontol 2000 1997;14: 216-248.

21. Salminen A, Gursoy UK, Paju S, et al. Salivary biomarkers of bacterial burden, inflammatory response, and tissue destruction in periodontitis. J Clin Periodontol 2014;41:442-450.

22. de Molon RS, de Avila ED, Boas Nogueira AV, et al. Evaluation of the host response in various models of induced periodontal disease in mice. JPeriodontol 2014; 85:465-477.

23. He J, Yang L, Qing Y, He C. Effects of electroacupuncture on bone mineral density, oestradiol level and osteoprotegerin ligand expression in ovariectomised rabbits. Acupunct Med 2014;32:37-42.

24. Crotti T, Smith MD, Hirsch R, et al. Receptor activator NF kappaB ligand (RANKL) and osteoprotegerin (OPG) protein expression in periodontitis. J Periodontal Res 2003;38:380-387.

25. Park SI, Sunwoo YY, Jung YJ, et al. Therapeutic effects of acupuncture through enhancement of functional angiogenesis and granulogenesis in rat wound healing. Evid Based Complement Alternat Med 2012;2012: 464586.

26. Lu T, Luo Y, Sun H, Qin W, Li Y. Electroacupuncture improves behavioral recovery and increases SCF/c-kit expression in a rat model of focal cerebral ischemia/ reperfusion. Neurol Sci 2013;34:487-495.

27. Qin Y, He J, Xia L, Guo H, He C. Effects of electroacupuncture on oestrogen levels, body weight, articular cartilage histology and MMP-13 expression in ovariectomised rabbits. Acupunct Med 2013;31:214-221.

28. Lee HJ, Kang IK, Chung CP, Choi SM. The subgingival microflora and gingival crevicular fluid cytokines in refractory periodontitis. J Clin Periodontol 1995;22: 885-890.

29. Balto K, Sasaki H, Stashenko P. Interleukin-6 deficiency increases inflammatory bone destruction. Infect Immun 2001;69:744-750.

30. Darowish M, Rahman R, Li P, et al. Reduction of particle-induced osteolysis by interleukin- 6 involves anti-inflammatory effect and inhibition of early osteoclast precursor differentiation. Bone 2009;45:661 668.

31. Båge $\mathrm{T}$, Kats $\mathrm{A}$, Lopez BS, et al. Expression of prostaglandin $\mathrm{E}$ synthases in periodontitis immunolocalization and cellular regulation. Am J Pathol 2011; 178:1676-1688.

32. Blackwell KA, Raisz LG, Pilbeam CC. Prostaglandins in bone: Bad cop, good cop? Trends Endocrinol Metab 2010;21:294-301.

33. Choi DC, Lee JY, Lim EJ, Baik HH, Oh TH, Yune TY. Inhibition of ROS-induced P38MAPK and ERK activation in microglia by acupuncture relieves neuropathic pain after spinal cord injury in rats. Exp Neurol 2012; 236:268-282.

34. Shi GX, Liu CZ, Zhu J, Guan LP, Wang DJ, Wu MM. Effects of acupuncture at Sanyinjiao (SP6) on prostaglandin levels in primary dysmenorrhea patients. Clin $J$ Pain 2011;27:258-261.

35. Zhang $\mathrm{H}$, Bian Z, Lin Z. Are acupoints specific for diseases? A systematic review of the randomized controlled trials with sham acupuncture controls. Chin Med 2010;5:1.

36. Uemura K, Li M, Tsutsumi T, et al. Efferent vagal nerve stimulation induces tissue inhibitor of metalloproteinase-1 in myocardial ischemia-reperfusion injury in rabbit. Am J Physiol Heart Circ Physiol 2007;293: H2254-H2261.

37. Gürgen SG, Sayın O, Cetin F, Tuç Yücel A. Transcutaneous electrical nerve stimulation (TENS) accelerates cutaneous wound healing and inhibits pro-inflammatory cytokines. Inflammation 2014;37:775-784.

38. Kim JH, Choi H, Suh MJ, Shin JH, Hwang MH, Lee HM. Effect of biphasic electrical current stimulation on IL1 beta-stimulated annulus fibrosus cells using in vitro microcurrent generating chamber system. Spine (Phila Pa 1976) 2013;38:E1368-E1376.

39. Oda M, Arihiro K, Kataoka T, Osaki A, Asahara T, Ohdan H. Comparison of immunohistochemistry 
assays and real-time reverse transcription-polymerase chain reaction for analyzing hormone receptor status in human breast carcinoma. Pathol Int 2010;60:305-315.

40. Lau WK, Lau YM, Zhang HQ, Wong SC, Bian ZX. Electroacupuncture versus celecoxib for neuropathic pain in rat SNL model. Neuroscience 2010;170:655-661.

41. Chen L, Zhang J, Li F, et al. Endogenous anandamide and cannabinoid receptor-2 contribute to electroacupuncture analgesia in rats. J Pain 2009;10:732-739.
Correspondence: Dr. Flávia Aparecida Chaves Furlaneto, Department of Oral and Maxillofacial Surgery and Periodontology, Ribeirao Preto School of Dentistry, University of São Paulo, Av. do Cafe, s/n 14040-940, Ribeirao Preto, São Paulo, Brazil. Fax: 55-16-3602-4788; e-mail: flafurlaneto@hotmail. com.

Submitted November 9, 2014; accepted for publication January 26, 2015. 